\title{
Inequality, Coalitions and Collective Action
}

\author{
Pranab K. Bardhan* \\ Nirvikar Singh ${ }^{+}$
}

May 2004

\begin{abstract}
In a model where cooperation is beneficial, but subject to cheating, and is supported by trigger strategy punishments in a repeated game, we explore the relationship between the nature of cooperation (size and composition of coalitions) and underlying inequality in the distribution of private productive assets.
\end{abstract}

JEL Codes: C72, D30, D70, O10, P0

Key Words: inequality, self-enforcing, collective action, infrastructure

\footnotetext{
${ }^{*}$ Department of Economics, University of California at Berkeley

${ }^{+}$Department of Economics, University of California at Santa Cruz. Address: Social Sciences 1 FacServ, 1156 High Street, Santa Cruz, CA 95064. Phone: 831-459-4093. Email: boxjenk@ucsc.edu.
} 


\section{Introduction}

In two fields of economics there has been some literature on the "common pool" problem, where there are multilateral incentives to defect from cooperative strategies.

One of these fields relates to groups drawing upon the common pool of public budgetary resources discussed in the context of fiscal stabilization primarily in Latin America (see, for example, Velasco (1998); the other relates to the collective action problem in management of local environmental resources (see, for example, Baland and Platteau (2003). In the former, primarily macro literature, the typical models assume symmetry among the players, so that the incentives are also symmetric. However, if players differ systematically in some initial conditions (for example, in their initial wealth), their incentives to defect may also differ systematically. This has implications for the size and composition of groups that are able to sustain cooperation, and this is the issue that we explore in this paper.

In the second, more micro, literature initial wealth inequality has been considered, but mostly in the context of static two-player games. In this paper we construct a repeatedgame, multi-player model (allowing for coalition formation), focusing on the effects of wealth inequality in the matter of collective action in management of local environmental resources or in firms or localities benefiting from a common provision of infrastructural services.

In a one-shot 'prisoners' dilemma' type situation, defection is always a dominant strategy. Hence, cooperation in equilibrium requires some additional device. Here we use the standard assumption of repeated interaction, with trigger strategies that support cooperation as a subgame perfect Nash equilibrium (SPNE).

Our model considers distributional inequality with respect to a nontransferable resource, which we simply call private capital. The non-transferability assumption is only an extreme form of capital market imperfection that in a milder form is not unrealistic in most situations. Furthermore, it considers a setting in which cooperation promises to yield efficiency gains relative to the status quo not by promoting efficiency-enhancing redistributions of capital (i.e., from where its marginal return is already low to where it is still high), but instead, by promoting the adoption of efficient technologies for producing 
capital-complementing inputs. Examples of such inputs could be R\&D, irrigation, infrastructural services, etc.

In our model, incentives for cooperation tend to be stronger for wealthier agents (all else equal) and there can be a threshold level of distributional inequality beyond which cooperation is enforceable for the richly endowed and unenforceable for the poorly endowed. This result is somewhat similar to that of common pool models in which cooperation is more easily sustained in times of plenty, due to the fact that wealth accumulation and incentives for maintaining cooperation are mutually reinforcing (Aizenman, 1998; Benhabib and Rustichini, 1996), although the logic of our model is quite different.

Our paper examines when different kinds of coalition structures can emerge, and what the impacts of redistribution may be in such settings. A key idea that we explore is that too much inequality destabilizes cooperation by making it impossible to satisfy some incentive compatibility constraints, particularly those of the poor. In some cases, redistribution that increases inequality may actually hurt people who directly gain from the redistribution, through this process of destabilizing cooperation. Conversely, redistribution that reduces inequality may help those who give up some wealth, by increasing the scope of cooperation.

The rest of the paper is structured as follows. Section 2 presents the model. Section 3 presents basic results for the case where the fruits of cooperation do not depend on the size or composition of the coalition. This section also considers the effects of ex post side payments. Section 4 allows for size and composition effects, and considers the welfare impacts of redistribution in more detail. Section 5 concludes.

\section{Model}

Consider the following infinitely repeated game among $n$ agents, indexed by $i$, from poorest to richest, in terms of their private capital, $K_{i}$. Hence, $K_{i} \leq K_{i+1}$. Time proceeds in discrete periods, and the agents have a common discount factor, $\beta$. 


\section{Stage Game}

The economy enters each new period with a total private capital endowment of $K$, of which each agent controls share $s_{i}$. Hence, $K_{i} \equiv s_{i} K$. Each agent's utility is assumed to be increasing in his consumption of own-produced output, $Y_{i}$, and decreasing in his effort level, $e_{i}$ with the linear from given by:

$U_{i}=Y_{i}-c e_{i}$

While comparative statics with respect to $c$ can be carried out, they are relatively straightforward, and it simplifies notation to assume that $c=1$, which we impose in the rest of the paper.

Production of output $Y_{i}$ is assumed to be described by a Cobb-Douglas technology, with constant returns to scale with respect to private capital $K_{i}$, and a complementary input, labeled $A_{i}$, which may represent infrastructure or something similar:

$Y_{i}=A_{i}^{\alpha} K_{i}^{(1-\alpha)}$.

Property rights with respect to private capital are assumed to be fully secure. The complementary input $A_{i}$ is produced by agent effort via one of two possible technologies, and can be either private or public (in the non-excludable sense) depending on the technology selected. Each agent has access to a unique 'status quo' technology, defined by the equation:

$A_{i}=R_{L i} e_{i}$

In most of the paper, we shall assume that $R_{L i}$ is the same for all individuals. However, we will also briefly analyze the case where $R_{L i} \leq R_{L i+1}$, so that a richer agent's status quo production technology may be superior to that of a poorer player. Instead of the individual status quo technology, the agents may also choose to employ a 'cooperative' technology that is defined by the equation

$\Sigma A_{i} \quad=R_{H} \Sigma e_{i}$

where $R_{H}>R_{L i}$ for all $i$. While the cooperative technology is the most efficient option, we assume that its output (measured in units of the complementary input $A$ ) is subject to a pure common pool problem - namely, each agent has the ability to grab all the fruits of 
cooperation, $R_{H} \Sigma e_{i}$, for himself. Again, later in the paper, we analyze what happens when this extreme assumption is relaxed.

In contrast, each agent is assured of securing the fruits of his own labor when using his status quo technology. As a result, use of the cooperative technology for production of the complementary input $A$ cannot be sustained in the absence of the appropriate enforcement mechanisms. Formally, we can represent the stage game as a prisoners' dilemma in which the only Nash Equilibrium involves mutual defection. The game is illustrated for the two-person case in Table 1, where the agents are labeled R and P for rich and poor. Note that in the general, n-person case, there will always be an incentive for any individual to deviate, for any subset of cooperating individuals. Hence, the uniqueness of Nash Equilibrium applies in general.

Table 1

\section{Payoff matrix (payoffs denoted in units of the production input $\boldsymbol{A}$ )}

\begin{tabular}{|c|c|c|c|}
\hline & \multicolumn{2}{|c|}{ Rich Agent } \\
\hline & & Cooperate & Defect \\
\hline \multirow{5}{*}{$\begin{array}{l}\text { Poor } \\
\text { Agent }\end{array}$} & \multirow{3}{*}{ Cooperate } & $R_{H} e_{R}$ & $R_{H}\left(e_{R}+e_{P}\right)$ \\
\hline & & $R_{H} e_{P}$ & 0 \\
\hline & & 0 & $R_{L R} e_{R}$ \\
\hline & \multirow[t]{2}{*}{ Defect } & & \\
\hline & & $R_{H}\left(e_{R}+e_{P}\right)$ & $R_{L P} e_{P}$ \\
\hline
\end{tabular}

As is evident from Table 1, we are making two simplifying assumptions: the first is that mutual defection yields payoffs equivalent to those obtained when each player simply chooses his status quo technology (although all that matters is that mutual defection generates individual payoffs strictly greater than zero, and strictly less than those from mutual cooperation); the second is that, in the fully cooperative outcome, the ratio of the players' payoffs is equal to the ratio of their contributions to the cooperative effort - 
namely, when both players cooperate, each player receives an amount of capitalcomplementing input equal to

$A_{i}=R_{H} e_{i}$

This assumption allows us to keep the algebra simple (in particular by allowing us to restrict the issue of free-riding to instances where an agent chooses to defect from the cooperative norm), but also has two important implications. First, as we can soon verify by checking equation (8), it yields the social surplus-maximizing distribution of the complementary input $A$, as the ratio $A_{i} / A_{j}$ is equal to $K_{i} / K_{j}$ in equilibrium ${ }^{1}$. Second, it implies that social welfare is independent of distributional inequality, and depends only on whether or not cooperation takes place (i.e., social welfare can take on only one of two possible values, one corresponding to the status quo outcome and one corresponding to the cooperative outcome).

\section{Stage game and lifetime payoffs}

In the decentralized equilibrium, each agent chooses $e_{i}$ to maximize equation (1), given equation (3). The solution to this optimization problem is

$$
e_{i, \text { statusquo }}=K_{i}\left(\alpha R_{L i}^{a}\right)^{\frac{1}{1-\alpha}}
$$

As is expected, each agent's optimal choice of effort is increasing in his holdings of private capital. $^{2}$ Substituting equation (6) into equation (1), and recalling that each agent has a discount factor of $\beta$, we can write the lifetime utility of each agent under the status quo as

\footnotetext{
${ }^{1}$ As can be easily verified, any equation of the form $S=A_{1}^{\alpha}{K_{1}}^{l-\alpha}+A_{2}{ }^{\alpha} K_{2}{ }^{l-\alpha}$, where the $A_{i}$ 's and $K_{i}{ }^{\prime}$ s must each sum to fixed quantities, is maximized when $A_{1} / A_{2}=K_{I} / K_{2}$.

${ }^{2}$ If we retain the cost of effort parameter, c, from equation (1), this would enter the denominator of the expression in equation (6), so that effort would be decreasing in the cost parameter.
} 


$$
Q_{i}=\frac{1}{1-\beta}\left[K_{i}\left(\alpha R_{L i}\right)^{\frac{\alpha}{1-\alpha}}(1-\alpha)\right]
$$

We now turn to the stage game payoffs under full cooperation. Since we have assumed that cooperation simply allows each agent to secure a return to effort equal to $R_{H}$ instead of $R_{L i}$ (equation (5) vs. equation (3)), each agent's effort choice under full cooperation is equal to:

$e_{i, \text { cooperate }}=K_{i}\left(\alpha R_{H}^{a}\right)^{\frac{1}{1-\alpha}}$

and we can express his lifetime utility under full cooperation as

$$
C_{i}=\frac{1}{1-\beta}\left[K_{i}\left(\alpha R_{H}\right)^{\frac{\alpha}{1-\alpha}}(1-\alpha)\right]
$$

From equations (7) and (9), it is clear that the rich agent does better than the poor agent in both the status quo and cooperative outcomes, and in addition, that the incremental payoff to cooperation relative to the status quo is higher for the rich agent than for the poor agent. Finally, let us calculate the stage game payoff from defecting when the other agent cooperates. Since a defecting agent $i$ appropriates the fruits of the effort of all other agents, $j$, the latter sum of efforts factors into his own effort choice. Given that all other agents $j$ cooperate, agent $i$ 's optimal choice of effort is equal to

$$
e_{i, \text { defect }}=K_{i}\left(\alpha R_{H}^{a}\right)^{\frac{1}{1-\alpha}}-\sum_{j \neq i} e_{j, \text { cooperate }}
$$

provided that this expression is nonnegative, revealing a free rider effect for the defecting player $i$. Thus, there is a double benefit from defection in this model: there is free riding in effort as well as all the fruits from cooperation being captured. Substituting equation (8) for $e_{j}$ into equation (10) yields

$$
e_{i, \text { defect }}=\operatorname{Max}\left\{0,\left(\alpha R_{H}^{\alpha}\right)^{\frac{1}{1-\alpha}}\left(K_{i}-\sum_{j \neq i} K_{j}\right)\right\}
$$

We see from equation (11) that, when defecting, an agent whose share of total private capital is no more than 0.5 chooses an effort level of zero, while an agent whose share of 
total private capital is greater than 0.5 - if such an agent exists - chooses a strictly positive effort level (equal to his cooperative effort level minus the cooperative effort level of all the other agents). Substituting equation (11) into equation (1), we can calculate the stage game payoffs from defection for these two types of agents, respectively, as

$$
D_{i}=K\left(\alpha R_{H}\right)^{\frac{\alpha}{1-\alpha}}\left(1-s_{i}\right)^{\alpha} s_{i}^{1-\alpha}
$$

and

$$
D_{i}=K\left(\alpha R_{H}\right)^{\frac{\alpha}{1-\alpha}}\left[s_{i}-\alpha\left(2 s_{i}-1\right)\right]
$$

An examination of equations (12) and (13) reveals that each type of player's static payoff from defection is a function of his share, $s_{i}$, of the total private capital. Consider equation (13) first, where $s_{i}>0.5$. Depending on the parameter $\alpha, D_{i}$ is monotonically increasing in the share $s_{i}$ if and only if $\alpha<0.5$. In other words, increasing the share of a dominant rich agent increases (decreases) static payoffs from defection when production relies more (less) heavily on private capital. Now consider equation (12). A simple calculation shows that $D_{i}$ is monotonically increasing in the share if and only if $\alpha<1-s_{i}$, where the quantity $\left(1-s_{i}\right)$ is greater than 0.5 , by assumption.

Therefore, whenever $\alpha<0.5$ (so that production is more private capital intensive), an increase in the share of private capital increases the defection payoffs of a dominant rich agent (if he exists) and decreases the defection payoffs of all other types of agents. On the other hand, whenever $\alpha>0.5$, the rich agent's defection payoffs are decreasing in his share, while the relationship between other agents' defection payoffs and their share is inverse U-shaped.

\section{Sustaining Cooperation}

We now identify the conditions under which trigger strategies can sustain cooperation in the infinitely repeated version of the stage game. Cooperation will be sustainable via trigger strategies if both players choose to cooperate with the understanding that 
defection at any time $t$ will trigger a permanent reversion to the Nash equilibrium of the stage game. That is, cooperation is sustainable so long as no agent at any time $t$ is willing to forsake the incremental returns to cooperation (relative to the status quo) from time $t+$ 1 onwards in exchange for the one-time payoff from defection at time $t$. Formally, cooperation is sustainable if and only if the condition labeled $\mathrm{IC}_{\mathrm{i}}$ holds:

$\mathrm{IC}_{i}: \quad C_{i} \geq D_{i}+\beta Q_{i}$

for each agent $i$.

The incentive compatibility constraint particular to this model is as follows for an agent whose share is no more than 0.5 :

$\mathrm{IC}_{i}: \frac{1}{1-\beta}\left[1-\beta\left(\frac{R_{L i}}{R_{H}}\right)^{\frac{\alpha}{1-\alpha}}\right] \geq \frac{1}{1-\alpha}\left(\frac{1-s_{i}}{s_{i}}\right)^{\alpha}$

It is as follows for an agent whose share exceeds 0.5 :

$\mathrm{IC}_{i}^{\prime}: \frac{1}{1-\beta}\left[1-\beta\left(\frac{R_{L i}}{R_{H}}\right)^{\frac{\alpha}{1-\alpha}}\right] \geq \frac{\alpha}{1-\alpha}\left(\frac{1-s_{i}}{s_{i}}\right)+1$

In either case, the left hand side of the condition is greater than 1 , since $R_{H}$ is greater than $R_{L i}$. As expected, either form of incentive compatibility constraint is more likely to hold when agents are more forward-looking ( $\beta$ is larger), and when the incremental return to cooperation relative to the status quo is larger $\left(R_{H}\right.$ is larger relative to the $\left.R_{L i}\right)$. We are particularly interested in the impact of changes in the shares, $s_{i}$, on the incentive compatibility condition. In order to investigate this, it is useful to combine (15) and (16) by defining the function $\phi(s, \alpha)$ as follows:

$$
\phi(s, \alpha)=\left\{\begin{array}{lll}
\frac{1}{1-\alpha}\left(\frac{1-s}{s}\right)^{\alpha} & \text { if } & s \leq 0.5 \\
\frac{\alpha}{1-\alpha}\left(\frac{1-s}{s}\right)+1 & \text { if } & s>0.5
\end{array}\right.
$$

The following Lemma will be very useful in our subsequent analysis. 


\section{Lemma 1}

$\phi(s, \alpha)$ is continuous and differentiable in $s$ at $s=0.5$. Furthermore, it is strictly decreasing for $0 \leq s \leq 1$.

\section{Proof}

When $s=0.5, \phi(s, \alpha)=1 /(1-\alpha)$. The function is clearly continuous from the left. Taking the limit as $s$ approaches 0.5 from the right, we get $\alpha /(1-\alpha)+1$, which equals $1 /(1-\alpha)$. This proves continuity.

The derivative of the function with respect to $s$ is given by

$$
\phi_{s}(s, \alpha)= \begin{cases}-\frac{\alpha}{1-\alpha}\left(\frac{1-s}{s}\right)^{\alpha-1}\left(\frac{1}{s^{2}}\right) & \text { if } \quad s<0.5 \\ -\frac{\alpha}{1-\alpha}\left(\frac{1}{s^{2}}\right) & \text { if } \quad s>0.5\end{cases}
$$

It is easy to check that the derivative function is continuous at $s=0.5$ (the left and right derivatives are equal). Hence $\phi(s, \alpha)$ is differentiable in $s$ at $s=0.5$. Finally, we see that the derivative is always negative for the specified domain of $s$, establishing that $\phi(s, \alpha)$ is strictly decreasing.

We are now ready to analyze the model and derive our results.

\section{Basic Results}

In this section, we maintain all the assumptions imposed so far, and add the assumption that $R_{L i}$ is independent of $i$. Looking at the incentive compatibility constraint, we see that this implies that the left hand side of that constraint is identical for all agents. The right hand side varies only as a result of varying shares of private capital. Hence, we will be able to derive some results that relate the formation of coalitions (cooperating with respect to producing the input $A$ ) to the distribution of private capital. 
Before proceeding, we rewrite the combined incentive compatibility constraint as follows:

$\mathrm{IC}_{i}: \frac{1}{1-\beta}\left[1-\beta\left(\frac{R_{L}}{R_{H}}\right)^{\frac{\alpha}{1-\alpha}}\right] \geq \phi\left(s_{i}, \alpha\right)$

It will also be useful to have a symbol for the left hand side of (18), and we shall denote this by $\Lambda$, where the dependence on the various parameters is suppressed for brevity. Note that our simplification implies that $\Lambda$ is the same for all agents.

We must note the following complication in looking at coalitions. Suppose that we consider any proper subset of the $n$ agents as a possible coalition. Then the total private capital of this subset will be lower than $\mathrm{K}$, and the shares of the agents in the potential coalition will be appropriately redefined. However, condition (18) still describes the incentive compatibility constraint faced by each member of the potential coalition, with the shares recalculated. For example, suppose that there are four individuals with shares $0.1,0.2,0.3$, and 0.4 . If the two poorest agents form a coalition, their shares in this group are now $1 / 3$ and $2 / 3$.

We begin by examining possible two person coalitions, where a coalition is our term for a group that cooperates in the production of infrastructure, as described earlier. First, we introduce the following definition.

\section{Definition 1}

A coalition is potentially stable if the incentive compatibility constraint (18) holds for each member of the coalition.

The reason we use the qualifier potentially is that satisfaction of (18) is necessary for a coalition to work, but it may not be sufficient to maintain the coalition, since there could be other, more attractive opportunities for combining with others. We discuss this issue after Proposition 4.

We begin with a preliminary result on two-person coalitions. 


\section{Proposition 1}

Assume that $\Lambda(1-\alpha)>1$. Consider the coalition of agents $i$ and $j$, with initial shares $s_{i}$ and $s_{j}$ of total private capital $K$, and assume, without loss of generality, that $s_{i}<s_{j}$. This coalition is potentially stable if and only if $s_{j} \leq(\Lambda(1-\alpha))^{\frac{1}{\alpha}} s_{i}$

\section{Proof}

From Lemma 1, we know that if (18) holds for $i$, it also holds for $j$. Hence, we need only check (18) for agent $i$, who must have a share less than 0.5. Therefore, using (17) and (18), we can rewrite the incentive compatibility condition as

$\Lambda(1-\alpha) \geq\left(\frac{1-\left(s_{i} /\left(s_{i}+s_{j}\right)\right)}{s_{i} /\left(s_{i}+s_{j}\right)}\right)^{\alpha}$

In doing so, we have calculated the coalition shares as discussed earlier in this section. This simplifies to $\Lambda(1-\alpha) \geq\left(\frac{s_{j}}{s_{i}}\right)^{\alpha}$

This can finally be rearranged as $s_{j} \leq(\Lambda(1-\alpha))^{\frac{1}{\alpha}} s_{i}$ which is the required condition.

\section{Remarks}

By assumption, the factor multiplying $s_{i}$ on the right hand side of the inequality condition in Proposition 1 is greater than 1, so that the inequality can potentially hold. The condition in Proposition 1 is clearly a combined assumption about the magnitudes of the discount factor, the relative gains from cooperation, and the importance of private capital in production. If it is violated, the two-person coalition can never be potentially stable. 
Even if it holds, the condition for potential stability requires that the inequality between the two partners be limited, as captured in the condition of Proposition 1. We can also note that, for any agent $i$, the case of minimal inequality for two-person coalitions involving this agent will be in partnership with either neighbor in the private capital distribution.

We can extend the analysis so far to larger coalitions, by noting the following negative result.

\section{Proposition 2}

If a two-person coalition involving agents $i$ and $j$ is not potentially stable, then adding other agents cannot make the resulting coalition potentially stable.

\section{Proof}

Adding other agents must reduce the shares of the agents in the new coalition versus the old. By Lemma 1, this can only increase the right hand side of (18). Hence, if (18) was violated for one of the agents in the two-person coalition, it must still be violated in the larger coalition.

Propositions 1 and 2 can be combined in the following result, which identifies when an individual cannot be part of any potentially stable coalition.

\section{Proposition 3}

If, for individual $i$, it is true that $s_{i+1}>(\Lambda(1-\alpha))^{\frac{1}{\alpha}} s_{i}$ and that $s_{i}>(\Lambda(1-\alpha))^{\frac{1}{\alpha}} s_{i-1}$, then this individual cannot be part of any potentially stable coalition.

\section{Proof}

By Proposition 1, we know that $i$ cannot be in a potentially stable two-person coalition with either of his neighbors in the distribution of private capital. Furthermore, he cannot be in a potentially stable two-person coalition with anyone else, since the inequality will 
only be greater. By Proposition 2, adding other agents to any two-person coalition containing $i$ will still leave at least one incentive compatibility condition unsatisfied.

\section{Remarks}

Proposition 3 tells us that inequality that is excessive anywhere in the distribution can isolate individuals, by making it impossible for them to engage in cooperative groups or coalitions.

Conversely, ruling out such cases allows one to characterize the potential for cooperation more sharply. We first define a coalition structure.

\section{Definition 2}

A coalition structure is a partition of the set $\mathrm{N}=\{1,2,3, \ldots, \mathrm{n}\}$, such that all the agents in any element of the partition cooperate with each other in production of the infrastructure good, and not with agents in any other element of the partition.

We have the following result on when $\mathrm{IC}_{i}$ (condition (18)) is satisfied for all agents except possibly the poorest, so that all coalitions in the structure are potentially stable.

\section{Proposition 4}

If $s_{i+1} \leq(\Lambda(1-\alpha))^{\frac{1}{\alpha}} s_{i}$ for all $i=1, \ldots n-1$, then there exists a coalition structure such that $\mathrm{IC}_{i}$ is satisfied at least for all $i>1$.

\section{Proof}

From condition (18), since the right hand side is strictly decreasing in $s_{i}$, if (18) holds for agent $i$, it must hold for all agents with index greater than $i$, by Lemma 1. Suppose that (18) holds for agent 1 . Then we are done, since (18) then holds for all agents. If not, then eliminate agent 1 , and recalculate the shares for everyone else as $s_{j}{ }^{\prime}=s_{j} /\left(1-s_{1}\right)$. If $s_{2}{ }^{\prime}$ is 
such that (18) holds, then again, by Lemma 1, it holds for all agents with index greater than 2 . If not, then we eliminate agent 2 and recalculate the shares.

Once we have reached the end of this iterative procedure, we have found an integer $m^{\prime}$ such that (18) holds for $i \geq m^{\prime}$. By Proposition 1, we know that $m^{\prime}<n$, since the coalition of the wealthiest two agents is potentially stable, by Proposition 1 . Now consider the remaining agents, with $i<m^{\prime}$. Recalculate the shares for this subset of agents. Once again, if (18) holds for agent 1 , then it holds for all agents in this subset. If not, eliminate agent 1 , recalculate the shares, and proceed as before. In each case, we must reduce $m^{\prime}$ by at least two at each stage. If at any stage, only agents 1 and 2 are left, then again, by Proposition 1, we know that they can form a potentially stable partnership. If only agent 1 is left, he may not be part of any potentially stable coalition, since, by construction, he cannot join any coalition formed at an earlier iteration.

Since satisfaction of the incentive compatibility condition (18) is the key necessary condition for a coalition of agents to engage in cooperation, Proposition 4 is the basis for characterizing one possible form of the equilibrium coalition structure. We need to make some observations and provide a definition before we proceed with this characterization. First, observe that an agent's utility from cooperation in the basic model is independent of the size and composition of the coalition to which he belongs: this is evident from equation (9). It follows that if an agent is part of any coalition, he has no incentive to deviate, either individually, or as part of a subgroup, as long as no ex post side payments are allowed. Furthermore, it follows that, in the absence of such side payments, an agent who is not part of any coalition cannot bribe an agent in a coalition to defect. With these points in mind, we provide the following definition.

\section{Definition 3}

A coalition structure is stable in the absence of side payments if no member of any coalition can gain by defecting in any of the following ways, where ex post side payments are not allowed: 
(i) unilaterally leaving one coalition to join another,

(ii) leaving the initial coalition together with others from the same coalition, to create a new coalition in which no one is worse off than before,

(iii) leaving the initial coalition together with others from different coalitions, to create a new coalition in which no one is worse off than before,

(iv) leaving the initial coalition together with others from the same coalition, to join an existing coalition in which no one in the enlarged coalition is worse off than before,

(v) leaving the initial coalition together with others from different coalitions, to join an existing coalition in which no one in the enlarged coalition is worse off than before.

We have offered an exhaustive list of possible deviations in our definition, on the basis of what seems reasonable. Note that deviations do not have to result in stable coalition structures, nor even be self-enforcing. Thus, we do not consider second order deviations, where, for example, some subset of those deviating will further deviate. Comparing this approach with that of the Coalition Proof Nash Equilibrium (CPNE) of Bernheim, Peleg and Whinston (1987), we are restricting the equilibrium more, by allowing more general deviations. The reason we are able to take this approach is the special structure of our model. Note that CPNE has been criticized by Xue (2000) on the basis of the nestedness and myopia assumptions that it incorporates. By relaxing both these, Xue offers an alternative equilibrium concept, that of Negotiation Proof Nash Equilibrium (NPNE). We relax the nestedness, but not the myopia assumption. Ray and Vohra (1997) offer an approach that is similar to CPNE, but allows for binding agreements. The difference that this makes to the analysis, as well as a clear and comprehensive discussion of the literature, can also be found in that paper. ${ }^{3}$

\footnotetext{
${ }^{3}$ It will be noticed that we also avoid any formal specification of bargaining procedures. A detailed analysis of bargaining protocols and coalition formation, and additional literature references, are both provided by
} 
With Definition 3, we can state our next result.

\section{Proposition 5}

If $s_{i+1} \leq(\Lambda(1-\alpha))^{\frac{1}{\alpha}} s_{i}$ for all $i=1, \ldots n-1$, then there always exists a coalition structure that is stable in the absence of side payments that has the following properties:

(i) Suppose that the elements of the partition, $P_{u}$, are ranked by the private capital of the richest agent in each element, so that if $u^{\prime}<u^{\prime \prime}$, then the richest agent in $P_{u^{\prime \prime}}$ is at least as rich as the richest agent in $P_{u^{\prime}}$. Then it is the case that the poorest agent in $P_{u^{\prime \prime}}$ is also at least as rich as the richest agent in $P_{u^{\prime}}$.

(ii) Each agent belongs to a cooperative group, except possibly the single poorest agent.

\section{Proof}

By Proposition 4, we can find a partition such as that described in the statement of Proposition 5, where each agent is part of a cooperative group, except possibly the poorest agent, and where $\mathrm{IC}_{i}$ is satisfied for each agent given the coalition structure. Since no single agent or group of agents can gain by defecting in any of the ways described in Definition 3, the result holds.

\section{Remarks}

The interest of Proposition 5 is that it describes a stable coalition structure that has the features of assortative matching equilibria (Becker 1973; Durlauf and Seshadri, 2003; the latter paper has additional references to this literature), but under very different

Ray and Vohra (1999). The special structure of our model includes the income distribution and production technology assumptions, as well as those with respect to moral hazard. Genicot and Ray (2003) analyze a model in which incentive constraints matter. In this paper, as well as in special cases considered in Ray and Vohra (1999), symmetry is used to sharpen the analysis. In contrast, asymmetry in the form of a wealth distribution is central to our analysis. 
conditions. In the basic model, there is no productivity gain to aligning with those who have similar wealth endowments. Instead, the logic of combining with those who are close in the wealth distribution is that it reduces inequality within the coalition, making it easier to satisfy the incentive compatibility constraints. This feature is not present at all in the usual assortative matching models.

Note also that the coalition structure in Proposition 5 need not be a unique equilibrium. This will be seen explicitly in Proposition 7, below.

The next result describes the impact of a particular kind of increase in inequality on the equilibrium described in Proposition 5.

\section{Proposition 6}

Given a stable coalition structure of the form described in Proposition 5, a redistribution of private capital that leads to a Lorenz-dominating increase in inequality within a group cannot increase the size of that group, and may reduce it, assuming that the form of the coalition structure does not change.

\section{Proof}

By Lemma 1, the poorest person in the group has the tightest incentive compatibility constraint. A Lorenz-dominating increase in inequality either reduces the poorest person's share, or leaves it unchanged. In the former case, if the share is reduced enough, the incentive compatibility condition is violated, and the poorest person may have to drop out of the coalition.

The condition used in Propositions 4 and 5, by ruling out the case analyzed in Proposition 3 , ensures that one does not have to have isolated individuals (i.e., those who are not part of any cooperative group in a stable coalition structure) in the middle of the wealth distribution. The equilibrium coalition structure described in Proposition 5 involves sorting by wealth levels. The next result shows that even if $s_{i+1} \leq(\Lambda(1-\alpha))^{\frac{1}{\alpha}} s_{i}$ for all $i=$ 
$1, \ldots n-1$, we may have non-assortative coalition structures that are stable. Note that the assortative structure must also be a possible equilibrium in such cases.

\section{Proposition 7}

Even if $s_{i+1} \leq(\Lambda(1-\alpha))^{\frac{1}{\alpha}} s_{i}$ for all $i=1, \ldots n-1$, there may exist a coalition structure that is stable in the absence of side payments in which individuals who are not adjacent in the wealth distribution cooperate, excluding those in between.

\section{Proof}

The proof is by construction.

Let $n=3$, and let $(\Lambda(1-\alpha))^{\frac{1}{\alpha}}=2$.

Let the shares be $0.25,0.3$ and 0.45 . We see that the condition of the proposition, $s_{i+1} \leq(\Lambda(1-\alpha))^{\frac{1}{\alpha}} s_{i}$, is easily satisfied. Furthermore, (18) is violated for agents 1 and 2 , so the grand coalition of all three agents is not potentially stable.

Now consider the coalition $\{1,3\}$. It is easy to check that $s_{3}{ }^{\prime} \leq(\Lambda(1-\alpha))^{\frac{1}{\alpha}} s_{1}{ }^{\prime}$, where these are the shares recalculated for the two-person coalition, and this is the IC condition for agent 1 (the IC condition for 3 is then satisfied, by Lemma 1). Hence the coalition is potentially stable. Hence the coalition structure $[\{1,3\},\{2\}]$ is stable.

\section{Remarks}

In the example, it is also the case, as was discussed above, that the coalition structure which has the form described in Proposition 5, i.e., $[\{1\},\{2,3\}]$, is stable. In fact, the structure $[\{1,2\},\{3\}]$ is also stable in this case. Also, note that in the example, $\left(1-s_{2}\right) / s_{2}>\Lambda(1-\alpha)^{\frac{1}{\alpha}} \geq s_{3}^{\prime} / s_{1}^{\prime}=s_{3} / s_{1}$. Taking the inequality between the two extreme expressions and simplifying, we get the necessary condition that $\left(1-s_{2}\right) s_{2}<s_{1}$ 
for the coalition $\{1,3\}$ to be potentially stable. The interest of this condition is that it puts a bound on local wealth inequality that is independent of the other parameters, unlike the condition used in Proposition 1.

The last inequality condition is specific to the three-person example. We can generalize it as follows. Let $\mathrm{T}$ be a proper subset of $\mathrm{N}$, and suppose agents $k, k+1$ are not in $\mathrm{T}$. Let $S_{T} \equiv \sum_{j \in T} s_{j}$. For simplicity, assume that the share of agent $k+1$ does not exceed 0.5 . If the IC condition is violated for agents $k, k+1$ in the coalition $\mathrm{T} \cup\{k, k+1\}$, but satisfied for agent $k$ in the coalition $\mathrm{T} \cup\{k\}$, then the following two inequalities must hold simultaneously:

$$
\begin{aligned}
& \left(S_{T \cup\{k, k+1\}}-s_{k+1}\right) / s_{k+1}>(\Lambda(1-\alpha))^{\frac{1}{\alpha}}, \text { and } \\
& \left(S_{T \cup\{k\}}-s_{k}\right) / s_{k} \leq(\Lambda(1-\alpha))^{\frac{1}{\alpha}} .
\end{aligned}
$$

Combining the inequalities and simplifying, we obtain the general necessary condition that $s_{k}>s_{k+1}\left(1-s_{k+1} / S_{T \cup\{k, k+1\}}\right)$. Again, this gives an upper bound on local inequality that is independent of the other parameters. If it is violated, we have the following negative result, which rules out coalitions in which a poorer person in the wealth distribution is included, but his richer distributional neighbor is not.

\section{Proposition 8}

Suppose $\mathrm{T}$ is any proper subset of $\mathrm{N}$, and suppose agents $k, k+1$ are not in $\mathrm{T}$. If, in the coalition $\mathrm{T} \cup\{k, k+1\}$, the share of $k+1$ does not exceed $0.5, k+1$ violates (18) for this coalition, and $s_{k} \leq s_{k+1}\left(1-s_{k+1} / S_{T \cup\{k, k+1\}}\right)$, then $\mathrm{T} \cup\{k\}$ cannot be a potentially stable coalition.

\section{Proof}

The proof follows immediately from the calculations preceding the proposition. 
The analysis to this point is greatly simplified by the fact that the size and composition of a cooperative group do not affect its productivity. In this case, as long as an agent is part of a coalition, the structure of the coalition does not matter. Given a stable coalition structure, the only agents who can benefit from a change in the structure are those who are isolated, and not part of any cooperative group. Since they benefit, they can potentially bribe someone to defect from their initial group and partner with them, but we have ruled out such ex post side payments up to this point. We first analyze the implications of relaxing this assumption in the basic model.

The possibility of side payments introduces a major complication if they are fully anticipated. Recall that an agent's effort choice under cooperation was assumed to be determined based on a particular split of the jointly produced input, as given in equation (5). This split was used in deriving the equilibrium cooperative effort level, given in (8). If an agent anticipates that some of the gains from cooperation are going to have to be paid ex post in order to ensure cooperation, this can affect his effort decision. We shall finesse this problem by assuming that this link does not exist in the agent's calculations, because he treats ex post side payments as lump sum transfers.

Consider now the circumstances under which side payments may change the equilibrium coalition structure. Clearly, this can happen only if there is an individual who is not part of any coalition in an initial candidate for the equilibrium. Such an individual, say $j$, gets $Q_{j}$ instead of $C_{j}$ (equations (7) and (9)) and can gain up to $C_{j}-Q_{j}$ by gaining a partner. Thus, up to this amount can be used as a side payment to gain a partner, say agent $k$. Note that we are measuring side payments as a present discounted value: this can be converted to a constant per period payment in practice.

A constraint on the possibility of using a side payment is that the partnership must be potentially stable. Hence, the conditions such as those in Proposition 1 must hold for the pair, $j$ and $k$. A complication is that the side payment can itself modify the incentive compatibility conditions and we will return to a fuller analysis of this point, after several other issues have been noted.

Note that switching to this coalition by other agents only makes satisfaction of the incentive compatibility constraints more difficult, since the shares of coalition members 
fall when new members are added. Furthermore, there is no gain in utility from a larger coalition. All these factors imply that we need only consider deviations of the first type, of those listed in Definition 3. There is one other possibility, however. If $j$ and $k$ were both isolated in the initial candidate equilibrium, they could not have formed a potentially stable coalition in the first place, and they can do so now only if the transfer payments change the incentive compatibility conditions.

We first assume that $k$ is part of another coalition in the initial situation. Now there are two possibilities to consider. Either $k$ is initially part of a pair, or part of a larger group. In the latter case, switching by $k$ does not cost the initial coalition anything. Therefore, they will not pay anything to retain $k$ in their coalition. Thus, any side payment $\varepsilon>0$ will be sufficient to get $k$ to switch into a partnership with $j$. The only role the side payment will play is in achieving incentive compatibility. On the other hand, if $k$ is initially part of a pair, then $j$ has to be able to outbid $k$ 's initial partner, say $l$. The condition for this would be $C_{j}-Q_{j}>C_{l}-Q_{l}$. From equations (7) and (9), we see that this is equivalent to $K_{j}>K_{l}$.

At this stage, we have to consider the impact of side payments on the incentive compatibility constraints. Let $T_{i}$ be the transfer payment (in present value terms) received by agent $i$, where $T_{i}$ may be negative as well as positive. Assuming that the payment is timed in a way that it is not received if defection occurs, then the incentive compatibility constraint is now

$\mathrm{IC}_{i}(\mathrm{~T}): \quad C_{i}+T_{i} \geq D_{i}+\beta Q_{i}$

Substituting and rearranging, we get the following. The incentive compatibility constraint is as follows for an agent whose share is no more than 0.5:

$\mathrm{IC}_{i}(\mathrm{~T}): \frac{1}{1-\beta}\left[1-\beta\left(\frac{R_{L}}{R_{H}}\right)^{\frac{\alpha}{1-\alpha}}\right] \geq \frac{1}{1-\alpha}\left(\frac{1-s_{i}}{s_{i}}\right)^{\alpha}-T_{i} K /\left[s_{i}\left(\alpha R_{H}\right)^{\frac{\alpha}{1-\alpha}}(1-\alpha)\right]$

It is as follows for an agent whose share exceeds 0.5 : 
$\mathrm{IC}_{i}^{\prime}(\mathrm{T}): \frac{1}{1-\beta}\left[1-\beta\left(\frac{R_{L}}{R_{H}}\right)^{\frac{\alpha}{1-\alpha}}\right] \geq \frac{\alpha}{1-\alpha}\left(\frac{1-s_{i}}{s_{i}}\right)+1-T_{i} K /\left[s_{i}\left(\alpha R_{H}\right)^{\frac{\alpha}{1-\alpha}}(1-\alpha)\right]$

In each case, we see that a positive transfer payment relaxes the incentive compatibility constraint, while a negative one tightens it. Thus, since the right hand side is decreasing in $s_{i}$ in the absence of side payments, the only case in which a side payment can achieve incentive compatibility in a two-person coalition (where it would not hold otherwise) is through a transfer from the richer partner to the poorer one.

Also, note that if the transfer payment is negative, then the right hand side is still decreasing in $s_{i}$. However, if the transfer payment is positive, the last term is increasing in $s_{i}$, so this property no longer necessarily holds. Note that, as usual, if these constraints are applied to a proper subset of $\mathrm{N}$, the shares and total private capital are interpreted as those for the subset. This introduces a further complication, in that the last term introduces a dependence of the side payment on the total private capital of the coalition.

Now consider again the problem of whether agent $j$ will switch from partnering $l$ to partnering $j$. We have assumed that the partnership $\{k, l\}$ does not require side payments for incentive compatibility. If $K_{k}>K_{j}>K_{l}$, then $j$ and $k$ are closer in the wealth distribution than $k$ and $l$, and therefore the incentive compatibility constraints must hold for the partnership $\{j, k\}$ without side payments. In this case, the amount that $j$ has to pay to get $k$ to switch is enough so that (i) $j$ 's incentive compatibility constraint is still satisfied, (ii) l's incentive compatibility constraint is not satisfied, and (iii) $j$ 's individual rationality constraint is still satisfied. The latter inequality is simply $C_{j}-Q_{j}+T_{j} \geq 0$. However, since $D_{j}>(1-\beta) Q_{j}$, it follows that if the incentive compatibility constraint (19) holds, then so does individual rationality, so (iii) is redundant. In fact, since agent $l$ 's incentive compatibility constraint is tighter than agent $j$ 's in a partnership with $k$, the side payment has to be just enough so that if $l$ tries to match it, he will violate his incentive compatibility condition.

We illustrate the above discussion with the three-person special case that was used to prove Proposition 7, which we now label Example 1. 


\section{Example 1}

In this case, $n=3$, and $(\Lambda(1-\alpha))^{\frac{1}{\alpha}}=2$, while the shares are $0.25,0.3$ and 0.45 . The grand coalition of all three agents is not potentially stable, but any two-person coalition is potentially stable. Thus, in the absence of side payments, we have three possible stable coalition structures. Now consider the structure $[\{1,3\},\{2\}]$. Agent 2 can make a side payment to agent 3 , in order to create the alternative structure, $[\{1\},\{2,3\}]$. The transfer payment required to achieve this must satisfy the condition that if agent 1 were to make the same side payment, his incentive compatibility constraint in a partnership with 3 would be violated. This requires

$$
\Lambda<\frac{1}{1-\alpha}\left(\frac{1-s_{1}^{\prime}}{s_{1}^{\prime}}\right)^{\alpha}-T K^{\prime} /\left[s_{1}^{\prime}\left(\alpha R_{H}\right)^{\frac{\alpha}{1-\alpha}}(1-\alpha)\right]
$$

Here $s_{1}{ }^{\prime}=5 / 14$. If we assume that $\alpha=0.5$, then $\Lambda=2 \sqrt{ } 2$. Substituting in these values, we get

$2 \sqrt{ } 2<2 \sqrt{ } 1.8-56 T K^{\prime} /\left(5 R_{H}\right)$, or

$-T>5 R_{H}(\sqrt{ } 2-\sqrt{ } 1.8) / 28 K^{\prime}$

In this case, $K^{\prime}$ is the private capital of the coalition $\{1,3\}$.

There remains one other issue to be discussed, which we shall do in the context of this example. We have argued that the coalition $\{2,3\}$ will form over $\{1,3\}$, because 2 can make a side payment to 3 that cannot be matched by 1 , because it would lead to 1 's incentive compatibility constraint being violated. While we assumed that $\{1,3\}$ was the initial coalition, the same argument would apply if $\{2,3\}$ were the starting point. A third possibility is that $\{1,2\}$ is the initial coalition. In this case, 3 could make a side payment to 1 or to 2 . Since a side payment to a poorer partner will relax the recipient's incentive compatibility constraint, the only issue is the satisfaction of 3's own incentive compatibility constraint in the partnership. Thus, from 3's perspective, 1 and 2 are equally good partners. In either case, an arbitrarily small side payment will ensure that agent 3 gets a partner. 
The conclusion of our discussion, in the context of the example, is therefore that, of the three coalition structures that are stable in the absence of side payments, only the coalition structure $[\{1\},\{2,3\}]$ is stable in the presence of side payments. Note that the grand coalition is still not stable, because agent 1 cannot credibly make side payments to the others, since his incentive compatibility constraint will not hold in that case. In the stable coalition structure, 2 can be replaced cheaply, whereas 2 has to outbid 1 to partner with 3 . However, it seems the side payment that 2 must make to 3 will depend on the sequence of proposals. ${ }^{4}$

In general, the flavor of the example and the preceding general discussion suggest the following result.

\section{Proposition 9}

Assume $s_{i+1} \leq(\Lambda(1-\alpha))^{\frac{1}{\alpha}} s_{i}$ for all $i=1, \ldots n$-1. In the presence of ex post lump sum side payments, no stable coalition structure can leave agent $i$ isolated, where $i>1$.

\section{Proof}

The proof proceeds along the lines of the discussion preceding Example 1. An isolated agent who is not the poorest can displace the poorest person from a coalition that has members who are below him in the wealth distribution, by offering a transfer payment. If no such coalition exists, then either $i=1$, or the person immediately below him in the wealth distribution is also isolated, and the two of them can cooperate.

\section{Group Size or Composition Effects}

We now return to the case where there are no side payments. We introduce the complications that the size of the cooperative group or its composition affects the

\footnotetext{
${ }^{4}$ This might be formalized, using the techniques of Ray and Vohra $(1997,1999)$ or Xue $(2000)$.
} 
productivity of the coalition. We begin with group size effects. In this case, suppose that $R_{H}=R_{H}\left(N_{u}\right)$, where $N_{u}$ is the size of group $u$ in an overall coalition structure. We will assume that the function is strictly increasing, so that a larger group is more productive. Also, for consistency, we define $R_{H}(1)$ to be $R_{L}$. Intuitively, there must be some underlying economies of scale that yield the outcome of increasing productivity, but we do not model them explicitly.

Obviously, the left hand side of (18) is now increasing in $R_{H}$, so the incentive compatibility conditions are easier to satisfy for members of a larger group, other things equal. Thus individuals who would otherwise be left out may now be included in a cooperative group. In Example 1, suppose that $(\Lambda(1-\alpha))^{\frac{1}{\alpha}}=3$ for a three-person group, rather than 2 for any pair. Then the grand coalition can form, since 1's incentive compatibility condition is now satisfied in this case.

We will illustrate this case further with a slightly different example, and examine the impact of redistribution of private capital. In the model considered in Section 3, redistribution would help those agents who were isolated, and could not be part of any potentially stable coalition, as in the case analyzed in Proposition 3. Narrowing the gap between such an individual and one of his neighbors would help him beyond any utility gains through redistribution, by increasing his productivity. Conversely, there could be redistributions that might destabilize cooperation by increasing inequality between a potentially stable pair. In the following example, we will see that there are similar complications when group size affects the productivity of cooperation.

\section{Example 2}

Let $\beta=0.9, \alpha=0.5, R_{L}=1$, and $R_{H}=N^{0.5}$.

Then $\Lambda=10\left(1-0.9 N^{-0.5}\right)$.

Let $\mathrm{N}=4$, with distribution of shares given by $[0.05,0.2,0.3,0.45]$. 
We can calculate that $(\Lambda(1-\alpha))^{\frac{1}{\alpha}}$ is 7.625 for a group of $4,5.769$ for a group of 3 , and 3.305 for a group of 2 .

It can then be checked that only the three richest people satisfy the incentive compatibility constraint for the grand coalition.

For the coalition $\{2,3,4\}$, the incentive compatibility constraint is tighter than for the richest pair, but it is satisfied for all three individuals, since for the poorest in this group, the relevant value is $\left(S_{T}-S_{2}\right) / s_{2}=0.75 / 0.2=3.75$. Hence, a stable coalition structure in this situation is given by $[\{1\},\{2,3,4\}]$. The alternative structure of $[\{1,2\},\{3,4\}]$ will not be stable, because 2, 3 , and 4 all benefit by 2 joining the richest pair, through the increased productivity of the bigger group. The structure $[\{2\},\{1,3,4\}]$ is not stable because 1's incentive compatibility constraint is still violated in joining 3 and 4. Similarly, the structure $[\{1,2,3\},\{4\}]$ is also not stable for the same reason. All other cases can also be ruled out, so $[\{1\},\{2,3,4\}]$ is the only stable structure.

Now suppose that there is a redistribution of private capital from 2 to 3 , so that the new shares are $[0.05,0.1,0.4,0.45]$. Now the original structure is no longer stable, because 2's incentive compatibility constraint is violated in the grand coalition. The only stable coalition structure is now $[\{1,2\},\{3,4\}]$.

The per-period utility from cooperation is given by $K_{i}\left(\alpha R_{H}\right)^{\frac{\alpha}{1-\alpha}}(1-\alpha)$. Normalizing $K=$ 1 , and using the values given for the other variables, this becomes $0.25 s_{i} N^{0.5}$. We can use this expression to compute the utility profiles for each of the two wealth distributions. In the first case, this profile is $[0.0125,0.0866,0.1299,0.1949]$. In the second case, the utility profile is $[0.0177,0.0354,0.1414,0.1591]$. We see that the richest person is actually hurt by this redistribution, because it destabilizes the larger group, and reduces the benefits of scale that were previously enjoyed. Agent 3 is also hurt in this manner, but is more than compensated for this loss with a large direct wealth gain. However, the nature of the example suggests that there can be cases where even the agent who is the beneficiary of a redistribution may be made worse off as a result of destabilizing the cooperative group, as we show in Example 3. Also, note that in the current example, total utility goes down as the result of the redistribution, from 0.4239 to 0.3536 . 


\section{Example 3}

We keep the other parameter values of Example 2, but now let the initial distribution of shares be $[0.04,0.15,0.35,0.46]$. We can see that the coalition $\{1,2\}$ is not potentially stable, whereas the coalition $\{2,3,4\}$ is potentially stable, since $\left(S_{T}-S_{2}\right) / s_{2}=0.81 / 0.15=$ 5.40. Once again, the only stable coalition structure is $[\{1\},\{2,3,4\}]$. Consider a small redistribution from agent 2 to 3 , so that the new distribution is $[0.04,0.13,0.37,0.46]$. Now, for the coalition $\{2,3,4\},\left(S_{T}-S_{2}\right) / s_{2}=0.83 / 0.13=6.38$, which is greater than $(\Lambda(1-\alpha))^{\frac{1}{\alpha}}$, and so the coalition is no longer potentially stable. At the same time, the redistribution makes the coalition $\{1,2\}$ potentially stable, so that the stable coalition structure is now $[\{1,2\},\{3,4\}]$.

The utility profiles can again be computed from the expression $0.25 s_{i} N^{0.5}$, and these are $[0.0100,0.0650,0.1516,0.1992]$ before the redistribution, and $[0.0141,0.0460,0.1308$, 0.1626] afterwards. Here, all except the poorest agent are hurt by the redistribution, and total utility also falls. In particular, however, note that the small redistribution toward agent 3 hurts him by removing the possibility of simultaneous cooperation with agents 2 and 4 , in a three-person group.

\section{Example 4}

In the previous two examples, there was a redistribution of private capital from a poorer to a richer person, increasing inequality and destabilizing some cooperation. We now consider redistribution that reduces inequality and increases the size of the cooperative group. Keeping the other parameters the same, let the distribution of shares be given by $[0.1,0.2,0.3,0.4]$. In this case, the grand coalition is still not potentially stable. There are two possible stable coalition structures, based on satisfying the incentive compatibility constraints. One is $[\{1\},\{2,3,4\}]$, while the other is $[\{1,2\},\{3,4\}]$. The utility profiles in the two cases are $[0.0250,0.0866,0.1299,0.1732]$ and $[0.0354,0.0707,0.1061$, 0.1414 ] respectively. Hence, once again, 2, 3 and 4 can all benefit from 2's defection to join the richest two agents. Thus, only the structure $[\{1\},\{2,3,4\}]$ is actually stable. 
Now consider a redistribution from the richest agent to the poorest, so that the distribution of shares becomes [0.13, 0.2, 0.3, 0.37]. Now the grand coalition is stable, since 1's incentive compatibility constraint is now satisfied for this group. Furthermore, the utility profile becomes $[0.0650,0.1000,0.1500,0.1850]$, and all agents are better off as a result of the redistribution, even the richest agent, who gives up some private capital.

We now turn to the case where group size does not matter, but the average private capital of the group affects productivity, so that $R_{H}=R_{H}\left(\bar{K}_{u}\right)$, where $\bar{K}_{u}$ is the average private capital of group $u$. Thus, there are no economies of scale (beyond the benefits of cooperation rather than going it alone), but working with someone who has greater private capital confers a benefit: we can think of this as a simple case of externality. For example, if the private capital is human capital, one may learn more from working with someone who is better endowed in this respect. In this case, being in a larger group provides no benefits per se.

In fact, we can say quite a bit more beyond the lack of benefits of larger groups. We have the following result. In order to do so, we introduce the notation $\Lambda(T)$ for the value of $\Lambda$ when the coalition is $T$.

\section{Proposition 10}

If $s_{i+1} \leq(\Lambda(\{i, i+1\})(1-\alpha))^{\frac{1}{\alpha}} s_{i}$ for all $i=1, \ldots n-1$, no three agents have the same private capital, and the productivity of a cooperative group depends only on the average private capital of the group then there is a unique stable coalition structure that consists of pairs, starting from the richest pair and going down. If $\mathrm{N}$ is odd, then the poorest person is isolated.

\section{Proof}

Suppose that the richest two agents in the distribution are in the same group, which contains more than two agents. Then, since any adjacent pair can form a potentially stable coalition, this pair can be better off by defecting and forming a potentially stable pair, 
since this will maximize the productivity of the group to which they belong. Note that, since now $\Lambda$ depends on $\bar{K}_{u}$, we have to take account of this effect on the incentive compatibility constraints. However, the inequality in the statement of the proposition guarantees that they hold for the pair.

If the richest two agents are in different groups, again they can both defect and combine in a potentially stable pair. Since everyone else's private capital is lower, adding any third person to the pair can only reduce productivity and utility. Now consider the remaining distribution. One can make exactly the same argument for this distribution: the richest two persons remaining are best off by combining in a pair. Repeating this logic, either we have $\mathrm{N} / 2$ cooperative pairs if $\mathrm{N}$ is even, or $(\mathrm{N}-1) / 2$ pairs when $\mathrm{N}$ is odd, with the poorest person isolated.

Finally, we consider the case where the productivity of cooperation has scale effects as well as positive externalities. For example, we may have $R_{H}=r_{H} \sum_{j \in T} K_{j}^{\gamma}$. Now it pays to be in a cooperative group that is larger as well as wealthier: it increases one's utility, as well as relaxing the incentive compatibility constraints. It is easy to see that both kinds of effects explored earlier in this section will be operative. There will be a tendency toward larger coalitions, and, holding size constant, ones made up of wealthier agents rather than poorer ones. One can easily construct examples such as Example 4 for this case as well.

\section{Conclusion}

This paper has formulated a simple model of cooperation that captures some of the incentive considerations that affect the joint provision of infrastructure that will be subsequently used by group members. We postulate that repeated interaction can sometimes overcome moral hazard problems, but we find that this possibility is limited by within-group inequality. Therefore, the nature and inequality of the initial wealth distribution can affect the degree to which cooperation can be sustained. In fact, we have developed some illustrative cases in which redistribution from the rich to the poor 
improves the welfare of the rich, by making it possible to sustain greater cooperation than was previously possible.

Clearly, our results are for a special model, but one that has appealing features. Given the complexity of analyzing coalition formation in full, abstract generality, some additional structure is required to derive definite results on the nature of equilibrium. Our modeling to focus on inequality contrasts our analysis with other special models of coalition formation, in which symmetry is important in obtaining characterization results. In fact, in our analysis, the particular asymmetry associated with differences in wealth is a central feature.

\section{References}

Aizenman, Joshua, "Fiscal Discipline in a Union," in Sturzenneger, Federico and Mariano Tommasi (eds.), The Political Economy of Reform, Cambridge, MA: MIT Press, 1998.

Baland J.-M. and J.-P. Platteau, "Institutions and the Efficient Management of Environmental Resources" in Maler Karl-Goran and Jeffrey R. Vincent (eds.), Handbook of Environmental Economics, Elsevier, Amsterdam, 2003.

Becker, G.: A Theory of Marriage: Part I. Journal of Political Economy 81, 813-846 (1973)

Benhabib, Jess and Aldo Rustichini, "Social Conflict and Growth," Journal of Economic Growth 1 (1996), 125-142.

Durlauf, Steven N., and A. Seshadri, "Is Assortative Matching Efficient?," Economic Theory, 21, 475-493 (2003)

Garance Genicot and Debraj Ray, "Group Formation in Risk-Sharing Arrangements," Review of Economic Studies 70, 87--113 (2003)

Ray, Debraj, and Rajiv Vohra, "Equilibrium Binding Agreements," Journal of Economic Theory, 73, 30-78 (1997) 
Inequality and Collective Action: Bardhan \& Singh, May 2004

Ray, Debraj, and Rajiv Vohra, "A Theory of Endogenous Coalition Structures," Games and Economic Behavior, 26, 286-336 (1999)

Velasco, Andres, "The Common Property Approach to the Political Economy of Fiscal Policy”, in Sturzenneger, Frederico and Mariano Tommasi (eds.),The Political Economy of Reform, MIT Press, Cambridge, MA, 1998.

Xue, Licun, 2000, "Negotiation Proof Nash Equilibrium," International Journal of Game Theory, 29 (3), 339-357. 